\title{
KDIGO nomenclature glossary for Pediatric Nephrology
}

\author{
Joseph Laycock ${ }^{1}$ (D) $\cdot$ Michel Baum ${ }^{2} \cdot$ Lesley Rees ${ }^{1,3}$
}

Received: 28 May 2020 / Accepted: 28 May 2020 / Published online: 18 August 2020

(C) IPNA 2020

\section{Introduction}

Following publication of the recent KDIGO nomenclature consensus conference report [1], this month's issue of Pediatric Nephrology features an executive summary from the organizers [2]. This summary is being published concurrently in various general and kidney specialty journals, and sets out the rationale for why a standardized glossary is an important step at this time. The glossary is also available on the KDIGO website [3].

As pediatricians, we are keenly aware of the need for clear and effective communication. Not only are the children we treat often anxious about being sick and in an unfamiliar hospital environment but also their family members are often understandably emotionally distressed. In both cases, explaining what is going on in a way they can understand is vital to ease anxiety and build trust, and using ambiguous, misleading, or sesquipedalian language at such a vulnerable time can be confusing and even counter-productive.

The English language evolved through confluence, and so we have "kidney" from Middle English, "renal" from Latin, and "nephro" from Greek. While all three may be used relatively interchangeably in the medical literature, when talking to a patient or family member, most only understand "kidney." We have a responsibility to be considerate in our communication.

As journal editors, we also have a responsibility to ensure the research we publish is presented using precise nomenclature and standardized definitions, so that our readers receive straightforward, translatable results. Also, the terminology we

Joseph Laycock

joseph.laycock@ucl.ac.uk

1 Pediatric Nephrology, UCL Great Ormond Street Institute of Child Health, UCL, London, UK

2 UTSouthwestern Medical Center, Dallas, TX, USA

3 Department of Paediatric Nephrology, Great Ormond Street Hospital for Children NHS Foundation Trust, London, UK use with each other will influence the language we use with patients and their families. We should avoid redundant, outdated, and nebulous terms.

As a journal, we welcome the positive stance that this KDIGO glossary represents in seeking to ensure clear and consistent communication between researchers and clinicians, which in turn will inform healthy and productive doctorpatient (and family) communication. We endorse using the glossary and in particular, we are encouraging all our authors to adopt the following aspects of this nomenclature as key standards.

\section{Key glossary standards adopted by Pediatric Nephrology}

\section{“Kidney," "renal," “nephro..."}

These three words are often used interchangeably: for example, we publish papers which use "kidney transplant" or "renal transplant," but as stated above, patients and their families are far more likely to understand "kidney." As such, for the sake of standardization, where it is reasonable, we should use the term "kidney" preferentially.

Of course, it is not always reasonable to exchange one term for another, such as in specific diseases (e.g., nephronophthisis) or anatomical terms (e.g., renal artery), societies (e.g., IPNA), or our indeed journal name.

However, in many of the most common usages: disease, __ transplantation, ___ failure, "kidney" is much easier for the majority of people to understand.

\section{Recommendation}

Where possible, the term "kidney" should be used preferentially when describing kidney disease and function, rather than "renal" or the prefix "nephro-." For example, "kidney transplant" rather than "renal transplant." 


\section{Replacing "end-stage" with "stage 5" or "failure"}

The term "end-stage kidney disease" is ambiguous and imprecise - someone with "end-stage" may live for many more years on dialysis or even decades following transplantation. Not only that, but as revealed by focus group research conducted for the consensus conference, the term "end-stage" is potentially damaging and demoralizing for patients, as it "causes fear of the unknown, provokes undue trauma, implies impending death" [1]. Consideration should be taken to use patient-centered wording.

Indeed, "end-stage kidney disease" is redundant, having been replaced in 2012 with a more accurate diagnostic description based upon kidney replacement therapy (KRT) as per the KDIGO classification of chronic kidney disease (CKD) [4].

The KDIGO glossary also suggests the option of replacing "end-stage kidney disease" with "kidney failure." There have been concerns over use of the term "failure," especially as it was previously used in "acute renal failure" when describing AKI - in that context, it was potentially ambiguous as the "failure" may be reversible. However, in CKD stage 5, it does more accurately reflect the status of a kidney with irreversible loss of function. "Kidney failure" is also less stigmatizing as it is the kidney that is failing, not the patient who is "end-stage."

In pediatrics, we do not tend to use CKD risk classes which define CKD in terms of both GFR (G1-5) and albuminuria (A1-3), i.e., CKD G5A3. Therefore, we suggest simplifying the glossary abbreviations here to reflect this: i.e., CKD 5.

\section{Recommendation}

"End-stage" should be replaced by the relevant KDIGO CKD classification using the following terms and suggested abbreviations:

\begin{tabular}{ll}
\hline Term & Abbreviation \\
GFR $<15 \mathrm{ml} / \mathrm{min} / 1.73 \mathrm{~m}^{2}$ & \\
$\quad$ CKD stage 5 & CKD 5 \\
$\quad$ Or kidney failure & KF \\
Kidney replacement therapy & KRT \\
CKD stage 5 treated by dialysis & CKD 5D \\
CKD stage 5 treated by kidney transplant & CKD 5T \\
KD stage 5 with replacement therapy & CKD 5D \& 5T \\
CKD stage 5 without replacement therapy & CKD 5 without KRT
\end{tabular}

\section{AKI classification}

The conference consensus was that the 2012 KDIGO AKI classification [5] should be used consistently in publications, as it harmonizes the preceding RIFLE and AKIN definitions. However, the report also includes the caveat that application of definitions and classifications to children is more complex:

The 2012 AKI and CKD KDIGO guidelines contained commentary about application of the definitions and classifications to children. For example, duration $>3$ months does not apply to infants with CKD due to hypoplastic or dysplastic kidneys, and thresholds for albuminuria, proteinuria, and GFR differ in infants compared to adults. For studies in infants, further specification is required regarding use of terms to describe acute and chronic kidney disease and kidney disease measures. [1]

Infants and young children have different normative values for GFR and markers of acute kidney injury than adults. For example, in infants, an acute $50 \%$ decrease in the normal GFR for age also indicates AKI since infants and young children inherently have a lower normal GFR than adults.

As such, we recommend that where possible AKI should be defined according to the KDIGO AKI classification; however, as long as the authors clearly define their criteria and definitions, we are still willing to consider other classifications. This may apply especially to long-term follow-up studies or papers from regions with less penetrance of up to date training and information.

\section{Recommendation}

The KDIGO AKI classification should be used where possible.

\section{Other parts of the glossary}

The glossary is extensive, featuring five sections: (1) Kidney function and disease, (2) Kidney failure, (3) Acute kidney diseases and disorders (AKD) and acute kidney injury (AKI), (4) Chronic kidney disease (CKD), and (5) Kidney measures.

While many parts are generally applicable, other parts are more specific to adults. For example, heat maps/CKD risk categories, which use a color-coded system to display CKD prognosis by GFR and albuminuria, are not in widespread use in pediatrics. Adults are also more likely than children to have posttransplant CKD stratifications, etc.

Although the term "acute kidney disease" (AKD) was proposed by the KDIGO AKI working group back in 2012 [5], it does not appear to have developed much traction, at least in pediatrics. AKD concerns kidney diseases that last for up to 3 
months, i.e., as opposed to $\mathrm{CKD}$, with $\mathrm{AKI}$ as a subcategory of AKD. Currently, we do not see much use of the term AKD in submissions, and await the planned update of the KDIGO AKI guideline, which may reconsider the definition and classification.

\section{Process}

- We will incorporate these key standards from the glossary into our "Submission Guidelines" for authors on our journal homepage https://www.springer.com/journal/467

- As not all authors will be aware of these guidelines, submissions will be checked for adherence to the guidelines. Corresponding authors will be advised of the nomenclature glossary and key standards at revision and encouraged to revise their text accordingly.

- Over time, increased awareness of and adherence to the key standards will promote consistency in the design, execution, and writing up of research.

\section{Conclusion}

Overall, this nomenclature will hopefully lead to a helpful consistency in how we report kidney diseases in children, with the knock-on effects of improved clarity and reduced confusion in communication between doctors and patients and their families.

It is an opportunity to be more precise in how we describe research and will benefit editors, authors, and clinicians, as well as patients and their families, and will likely evolve over time. This idea will likely be taken up in other medical specialty fields.

\section{Compliance with ethical standards}

Conflict of interest The authors declare that they have no conflict of interest.

\section{References}

1. Levey A, Eckardt K, Dorman N, Christiansen S, Hoorn E, Ingelfinger J, Inker L, Levin A, Mehrotra R, Palevsky P, Perazella A, Tong A, Allison S, Bockenhauer D, Briggs J, Bromberg J, Davenport A, Feldman H, Fouque D, Gansevoort R, Gill J, Greene E, Hemmelgarn B, Kretzler M, Lambie M, Lane P, Laycock J, Leventhal S, Mittelman M, Morrissey P, Ostermann M, Rees L, Ronco P, Schaefer F, St. Clair Russell J, Vinck C, Walsh S, WeinerD CM, Jadoul M, Winkelmayer W (2020) Nomenclature for kidney function and disease: report of a kidney disease: improving global outcomes (KDIGO) consensus conference. Kidney Int 97: 1117-1129. https://doi.org/10.1016/j.kint.2020.02.010

2. Levey AS, Eckardt KU, Dorman NM, Christiansen SL, Cheung M, Jadoul M, Winkelmayer WC (2020) Nomenclature for kidney function and disease: executive summary and glossary from a kidney disease: improving global outcomes (KDIGO) consensus conference. Pediatr Nephrol. https://doi.org/10.1111/tri.13627

3. https://kdigo.org/conferences/nomenclature/. Accessed 25 May 2020.

4. Kidney Disease: Improving Global Outcomes (KDIGO) (2013) KDIGO 2012 clinical practice guideline for the evaluation and management of chronic kidney disease. Kidney Int Suppl 3:1-150

5. Kidney Disease: Improving Global Outcomes (KDIGO) Acute Kidney Injury Work Group (2012) KDIGO clinical practice guideline for acute kidney injury. Kidney Int Suppl 2:1-138

Publisher's note Springer Nature remains neutral with regard to jurisdictional claims in published maps and institutional affiliations. 